\title{
Walking behaviors for stroke survivors: comparison between straight line and curved path
}

\author{
Wonjeong Hwang ${ }^{a}$, Bora Choi ${ }^{b}$, Sujin Hwang ${ }^{c}$ \\ ${ }^{a}$ Research Facility of Academy of Women's Health in Physical Therapy, Sahmyook University, Seoul, Republic of Korea \\ ${ }^{\mathrm{b}} \mathrm{Ba}$ Reun Movement Exercise Center, Seoul, Republic of Korea \\ 'Department of Physical Therapy, Division of Health Science, Baekseok University, Cheonan, Republic of Korea
}

Objective: The purpose of this study was to compare walking conditions (straight line and curved path) on walking patterns in persons who had experienced hemiplegic stroke and to determine whether if they adapt their walking pattern and performances according to changes in environmental conditions.

Design: Cross-sectional study.

Methods: Forty-four hemiplegic stroke survivors participated in this study. This study measured walking performance in three different walking conditions, such as straight walking, the more-affected leg in the inner curve walking, and less-affected leg in the inner curve walking conditions, and a 2-dimentional gait analysis system was used as a primary measurement. This study also measured secondary clinical factors including the Timed Up-and-Go Test, the Trunk Impairment Scale, and the Dynamic Gait Index.

Results: After analyzing, cadence and step length of the less-affected side, stride length in the more-affected side, and stride length in less-affected side were significantly different among the three different walking conditions in this study $(p<0.05)$, but other temporospatial parameters were not significant. Cadence was the largest in the straight walking condition. Step length in the less-affected side, stride length in the more-affected side, and stride length in less-affected side were also the longest in the straight walking condition.

Conclusions: The results of the study suggest that hemiplegic stroke survivors show walking adaptability according to changes in walking demands and conditions, and moreover, cadence and step and stride lengths were significantly different between straight and curved walking conditions.

Key Words: Kinematics, Stroke, Walking

\section{Introduction}

Hemiplegia, a paralysis of one side of the body, occurs with stroke involving a sudden, specific neurovascular accident of the brain and the person is left with severe neurological and functional deficits. Hemiplegic stroke survivors have movement problems that lead to activity and participation limitations [1]. These movement problems manifest themselves as movement deficits in the trunk and extremities caused by missing pieces of movement control, atypical patterns of movement deviated from normal coordinated movement and undesirable compensations that lead to compensatory functional strategies [1]. These impairments ultimately interfere with normal functional movements and may lead to loss of independence in their daily life.

Walking patterns for hemiplegic stroke survivors are characterized by slow speed, uneven and decreased step and stride lengths, impaired balance and smooth translation of the body segment $[2,3]$. In particular, stroke survivors are impaired in their ability to produce automatic, reproducible

Received: 2 September, 2019 Revised: 25 September, 2019 Accepted: 26 September, 2019

Corresponding author: Sujin Hwang (ORCID https://orcid.org/0000-0001-8471-0103)

Department of Physical Therapy, Division of Health Science, Baekseok University, 76 Munam-ro, Dongnam-gu, Cheonan 31065 , Republic of Korea Tel: 82-41-550-2309 Fax: 82-41-550-2829 E-mail: sujin928@gmail.com

(c) This is an Open-Access article distributed under the terms of the Creative Commons Attribution Non-Commercial License (http://creativecommons.org/licenses/ by-nc/4.0) which permits unrestricted non-commercial use, distribution, and reproduction in any medium, provided the original work is properly cited.

Copyright (๑) 2019 Korean Academy of Physical Therapy Rehabilitation Science 
and integrated walking while adapting to the changes of environmental conditions. Walking performance for stroke survivors is a prerequisite for performing a safe recovery of activities and participation restrictions and therefore, it is necessary to understand the gait characteristics in various environmental demands within the rehabilitation setting [1,4-6].

This study aimed to simplify the characteristics of the kinematic parameters that was measured during three different walking performances, such as linear walking, the more-affected side in the inner curve walking, and the less-affected side in the inner curve walking conditions, as well as to determine whether they adapt their walking pattern according to changes in walking demands. We hypothesized that the hemiplegic stroke survivors will adapt and walk regardless of the walking paths aside from their gait abnormality, such as gait asymmetry, decreased stance phase, prolonged swing phase, and decreased walking speed.

\section{Methods}

A 2-demensional gait analysis system that was equipped with a walkway was designed to measure and to distinguish hemiplegic gait from normal gait. The study was approved by the Institutional Review Board (IRB) of Baekseok University and all participants provided their informed consent prior to the study (IRB No. BUIRB-201806-HR-004).

\section{Participants}

Forty-four stroke survivors who had a first diagnosis of stroke that was confirmed with neuroimaging 6 months ago or more (males and females, mean aged years) were includeed in the study. This study recruited persons who were able to walk $10 \mathrm{~m}$ or more independently $10 \mathrm{~m}$ with or without a gait aid on even surfaces, who had a Modified Ashworth Scale score of $<2$ in any of the hip, knee or ankle muscles, who had a Mini-Mental State Examination score of $>18$, and were independently walking prior to the first stroke accident. Subjects were excluded if they had unilateral neglect, recurrent stroke, uncontrolled hypertension, a history of other neurological impairments, and other factors such as impaired vision, vestibular deficits, cerebellar ataxia except stroke, and any musculoskeletal impairments that could affect gait performance.

\section{Experimental procedure}

This study measured primarily the temporospatial param- eters when the participants performed independent walking during three different walking conditions: straight line, the more-affected side in the inner curve walking, and the less-affected side in the inner curve walking. First of all, a 10 -m pedestrian walk was used for the straight walking condition and a pedestrian walk with a length of $10 \mathrm{~m}$ and a radius of $5 \mathrm{~m}$ was used for the curved walking conditions. The participants underwent gait performance under three different conditions. The walking conditions of the participants were randomly selected and all evaluations were conducted by experienced physical therapists who conducted clinical evaluation for 5 years or more. Each condition was performed five times and the average value was used for data analysis.

Demographic and clinical characteristics were obtained from the participants, medical records, or from performancebased examination and questionnaire-based examination.

\section{Experimental equipment and clinical measurement tools}

GAITRite (CIR Systems, Inc., Clifton, NJ, USA) is a truly portable single layer pressure sensitive walkway measuring temporal and spatial parameters and provides easy identification of gait performance via an electronic walkway. The study measured temporospatial parameters, such as gait velocity (obtained after dividing the distance by the ambulation time), cadence (the rate or steps per minute), step time (the time elapsed from the first contact of one foot to the first contact of the opposite foot), step length (measured on the horizontal axis of the walkway from the heel point of the current footfall to the heel point of the previous footfall on the opposite foot), and stride length (measured on the line of progression between the heel points of two consecutive footfalls of the same foot).

The Timed Up-and-Go test (TUG) is a simple clinical evaluation method used to assess the mobility of individuals who require both static and dynamic postural stability. In general, normal scores are less than 10 seconds for healthy adults, 11-20 seconds for those who are elderly or with disabilities, and more than 20 seconds indicates that assistance is required for outdoor walking and that additional tests and therapeutic interventions are needed. In addition, it is judged that there is a risk of falling if the TUG score is 30 seconds [7-9].

The trunk impairment scale (TIS) is an evaluation tool developed in 2004 by Verheyden et al. [10,11] for the purpose of evaluating the trunk function of stroke survivors. TIS evaluates static and dynamic sitting balance and trunk coor- 
dination in a sitting position for stroke survivors. Dynamic gait index is a clinical assessment tool that evaluates the ability to apply gait to complex gait tasks in everyday life. It evaluates gait ability in eight different demands and walking environments. Scores of 19 or less have been related to increase incidence of falls $[12,13]$.

\section{Data analysis}

All statistical analyses of the study were performed using PASW Statistics for Windows, Version 18.0 (SPSS Inc., Chicago, IL, USA). Descriptive statistics were used to describe demographic characteristics. The clinical characteristics are presented in Table 1 . To analyze the difference among three walking conditions, this study used variance assumptions with repeated measures with assumption of sphericity, which states that the variances within each walking conditions of difference scores. The degrees of freedom for the F-ratio was adjusted by multiplying them by a correc-

Table 1. Demographics of subjects

$(\mathrm{N}=44)$

\begin{tabular}{lc}
\hline \multicolumn{1}{c}{ Variable } & Value \\
\hline Sex (male/female) & $23 / 21$ \\
Age (y) & $61.30(13.11)$ \\
Post-duration disease (mo) & $32.14(29.43)$ \\
Etiology (infarction/hemorrhage) & $21 / 23$ \\
Brunnstrom's stage (4/5/6) & $20 / 20 / 4$ \\
Walking aid (with/without) & $15 / 29$ \\
Paretic side (right/left) & $24 / 20$ \\
Mini-mental state examination (score) & $26.27(3.19)$ \\
Timed up-and-go test (seconds) & $22.42(15.15)$ \\
Trunk impairment score (score) & $14.57(3.35)$ \\
Dynamic gait index (score) & $15.18(6.05)$ \\
\hline
\end{tabular}

Values are presented as number only or mean (SD). tion factor given the symbol epsilon, the GreenhouseGeisser correction. This study also used the Bonferroni as a post-hoc test. The significance level was set to $\alpha=0.05$.

\section{Results}

After analysis, cadence, step length of the less-affected side, stride length of the more-affected side, and stride length of the less-affected side were significantly different among the three different walking conditions in this study (Table 2). Cadence was significantly different between the straight line and the less-affected side in the inner curve walking conditions $(p=0.025)$. Step length in the less-affected side was significantly different between the straight line and the more-affected side in the inner curve and between straight line and less-affected side in the inner curve conditions ( $p=0.005)$. Stride length in the more-affected side was significantly different between the straight line and the more-affected side in the inner curve condition and between the more-affected side in the inner curve and less-affected side in the inner curve conditions $(p=0.024)$. Stride length in the less-affected side was significantly different between straight line and less-affected side in the inner curve walking conditions $(p=0.009)$.

\section{Discussion}

Independent, functional, and safe walking is difficult to retrain in the early phases following stroke due to impairment of trunk and extremity control, muscle weakness linked with trunk and extremity movements, asymmetrical weight bearing, atypical complex movement patterns, and poor postural stability [2]. Nevertheless, walking perform-

Table 2. Temporospatial parameters under three different walking conditions

$(\mathrm{N}=44)$

\begin{tabular}{lcccc}
\hline \multicolumn{1}{c}{ Variable } & Straight line & $\begin{array}{c}\text { More-affected side } \\
\text { in inner curve }\end{array}$ & $\begin{array}{c}\text { Less-affected side } \\
\text { in inner curve }\end{array}$ & $p$-value \\
\hline Gait velocity (cm/s) & $65.49(36.05)$ & $64.45(34.00)$ & $64.13(33.59)$ & 0.551 \\
Cadence (steps/min) & $88.52(24.89)$ & $87.45(25.27)$ & $86.76(25.68)^{\mathrm{a}}$ & 0.025 \\
Step time in more-affected (s) & $1.07(1.73)$ & $0.82(0.36)$ & $0.86(0.41)$ & 0.345 \\
Step time in less-affected (s) & $0.74(0.39)$ & $0.75(0.46)$ & $0.82(0.65)$ & 0.344 \\
Step length in more-affected (cm) & $42.99(13.42)$ & $40.59(14.16)$ & $43.05(12.87)$ & 0.136 \\
Step length in less-affected (cm) & $42.14(15.53)$ & $40.92(15.38)^{\mathrm{a}}$ & $40.27(15.74)^{\mathrm{a}}$ & 0.005 \\
Stride length in more-affected (cm) & $85.88(27.98)$ & $81.66(26.29)^{\mathrm{a}}$ & $84.53(27.09)^{\mathrm{b}}$ & 0.024 \\
Stride length in less-affected (cm) & $85.87(28.07)$ & $84.21(27.60)$ & $83.63(27.05)^{\mathrm{a}}$ & 0.009 \\
\hline
\end{tabular}

Values are presented as mean (SD).

${ }^{a}$ Significantly different compared with straight line. ${ }^{\mathrm{b}}$ Significant different compared with more-affected side. 
ance is necessary to maintain most of daily and functional activities for daily living and therefore the goal in a rehabilitation setting is to restore the walking performance in the earlier stages of stroke. The surrounding contexts of walking performance is not only always linear but also diverse, such as curved paths, slopes, stairs, and uneven surfaces.

During the usual straight line walking, spatiotemporal variabilities have been known to be an early indicator of mobility impairment and associated with risk of falls. Stroke survivors show decreased gait velocity, asymmetric walking pattern, and increased step width, and thereby show a different walking pattern compared to before the stroke event [14]. Spastic or synergistic gait patterns are atypical movement patterns in hemiparetic stroke patients and include contact with ground via toe strike or foot flat, loss of hip extension, and usage of excessive forward trunk flexion to initiate [1]. Forward progression instead of moving the shank and lower leg over the foot in stance phase, and proximal initiation patterns of pelvic hiking or rotation toward the affected side, hip flexion, with internal rotation and knee extension, or pelvic posterior tilting with hip abduction and knee flexion in swing phase are also shown [15]. However, a previous study reported that walking along a curved path implies a different spatiotemporal parameter of muscle activation compared with straight walking in humans [16-19]. After the studies, several researchers have reported on curved walking in hemiparetic individuals [20-22].

Godi et al. [20] reported the velocity of curved walking was not significantly smaller in hemiparetic patients compared with linear walking. Two other studies examined the neuromuscular strategies in the paretic leg during curved walking for persons with stroke $[21,22]$. Indeed, there is a lack of studies comparing linear walking and curved path walking in stroke surivors. This study evaluated the spatiotemporal parameters during straight walking and curved paths with the inner paretic side and the inner non-paretic side for stroke survivors. As of the results of a study by Godi et al. [20], the results of this study showed that gait velocity of curved walking was not significantly different from the linear walking speed. This study showed that three other spatiotemporal parameters (cadence, step length, and stride length) were significantly different between straight walking and curved path walking. When humans walk, if the external environment is changed, human walking is characterized by changing the number of steps or the step or stride lengths in order to maintain walking speed. This ability is namely gait adaptability or walking adaptability [23,24]. The results of this study suggest that one of reason why there was no significant difference in walking speed between straight walking and curved walking was walking adaptability. Therefore, gait adaptability should be considered when gait training for stroke survivors in rehabilitation settings.

This study was a cross-sectional, observational study design, not an experimental study. Future study should consider strategies to improving walking adaptability for stroke survivors. This study involved only one condition of curved walking and therefore, future studies require more severe curved walking conditions as well as uneven walking surface conditions.

\section{Conflict of Interest}

The authors declared no potential conflicts of interest with respect to the authorship and/or publication of this article.

\section{References}

1. Umphred DA. Umphred's neurological rehabilitation. 6th ed. St. Louis, Mo.: Elsevier/Mosby; 2013.

2. O'Sullivan SB, Schmitz TJ, Fulk GD. Physical rehabilitation. 6th ed. Philadelphia: F.A. Davis Company; 2014.

3. Dobkin BH. Clinical practice. Rehabilitation after stroke. N Engl J Med 2005;352:1677-84.

4. Lee J, Park S, Shin H. Detection of hemiplegic walking using a wearable inertia sensing device. Sensors (Basel) 2018;18:E1736.

5. Roth EJ, Merbitz C, Mroczek K, Dugan SA, Suh WW. Hemiplegic gait. Relationships between walking speed and other temporal parameters. Am J Phys Med Rehabil 1997;76:128-33.

6. von Schroeder HP, Coutts RD, Lyden PD, Billings E Jr, Nickel VL. Gait parameters following stroke: a practical assessment. J Rehabil Res Dev 1995;32:25-31.

7. Bonnyaud C, Pradon D, Vaugier I, Vuillerme N, Bensmail D, Roche N. Timed Up and Go test: Comparison of kinematics between patients with chronic stroke and healthy subjects. Gait Posture 2016;49:258-63.

8. Nordin E, Lindelöf N, Rosendahl E, Jensen J, Lundin-Olsson L. Prognostic validity of the Timed Up-and-Go test, a modified Get-Up-and-Go test, staff's global judgement and fall history in evaluating fall risk in residential care facilities. Age Ageing 2008;37:442-8

9. Bohannon RW. Reference values for the timed up and go test: a descriptive meta-analysis. J Geriatr Phys Ther 2006;29:64-8.

10. Verheyden G, Nieuwboer A, Mertin J, Preger R, Kiekens C, De Weerdt $\mathrm{W}$. The Trunk Impairment Scale: a new tool to measure motor impairment of the trunk after stroke. Clin Rehabil 2004; 18:326-34

11. Verheyden G, Nieuwboer A, Feys H, Thijs V, Vaes K, De Weerdt 
W. Discriminant ability of the Trunk Impairment Scale: a comparison between stroke patients and healthy individuals. Disabil Rehabil 2005;27:1023-8.

12. Hall CD, Schubert MC, Herdman SJ. Prediction of fall risk reduction as measured by dynamic gait index in individuals with unilateral vestibular hypofunction. Otol Neurotol 2004;25:74651 .

13. Jonsdottir J, Cattaneo D. Reliability and validity of the dynamic gait index in persons with chronic stroke. Arch Phys Med Rehabil 2007;88:1410-5.

14. Kao PC, Dingwell JB, Higginson JS, Binder-Macleod S. Dynamic instability during post-stroke hemiparetic walking. Gait Posture 2014;40:457-63.

15. Perry J, Burnfield JM. Gait analysis: normal and pathological function. 2nd ed. Thorofare: Slack; 2010.

16. Courtine G, Schieppati M. Human walking along a curved path. II. Gait features and EMG patterns. Eur J Neurosci 2003;18: 191-205.

17. Courtine G, Schieppati M. Human walking along a curved path. I. Body trajectory, segment orientation and the effect of vision. Eur J Neurosci 2003;18:177-90.

18. Courtine G, Schieppati M. Tuning of a basic coordination pattern constructs straight-ahead and curved walking in humans. J Neurophysiol 2004;91:1524-35.

19. Welch SA, Ward RE, Kurlinski LA, Kiely DK, Goldstein R, VanSwearingen J, et al. Straight and curved path walking among older adults in primary care: associations with fall-related outcomes. PM R 2016;8:754-60.

20. Godi M, Nardone A, Schieppati M. Curved walking in hemiparetic patients. J Rehabil Med 2010;42:858-65.

21. Duval K, Luttin K, Lam T. Neuromuscular strategies in the paretic leg during curved walking in individuals post-stroke. J Neurophysiol 2011;106:280-90.

22. Chisholm AE, Qaiser T, Lam T. Neuromuscular control of curved walking in people with stroke: case report. J Rehabil Res Dev 2015;52:775-83

23. Timmermans C, Roerdink M, van Ooijen MW, Meskers CG, Janssen TW, Beek PJ. Walking adaptability therapy after stroke: study protocol for a randomized controlled trial. Trials 2016;17: 425.

24. Balasubramanian CK, Clark DJ, Fox EJ. Walking adaptability after a stroke and its assessment in clinical settings. Stroke Res Treat 2014. doi: 10.1155/2014/591013 [Epub ahead of print] 